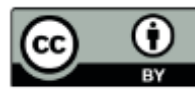

\title{
Las promotoras rurales de salud: una práctica social en extinción. Estudio de caso en el municipio de Andes, Antioquia, 1976-2015*
}

\author{
Rural Health Promoters: A Social Practice in Extinction. A Case \\ Study in the Town of Andes, Antioquia, 1976-2015
}

\section{Promotoras rurais de saúde: uma prática social em extinção. Estudo de caso no município de Andes, Antioquia, 1976-2015}

Recepción: 17 de diciembre de 2018. Aceptación: 28 de octubre de 2019.

Publicación: 30 de junio de 2020.

DOI: https://doi.org/10.11144/Javeriana.rgps19.prsp

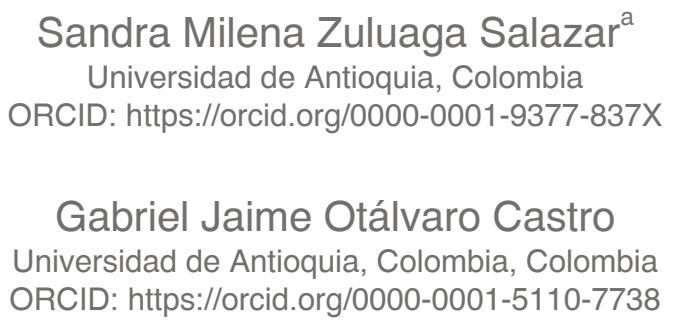

Cómo citar este artículo: Zuluaga-Salazar SM, Otálvaro-Castro GJ. Las promotoras rurales de salud: una práctica social en extinción. Estudio de caso en el municipio de Andes, Antioquia, 1976-2015. Revista Gerencia y Políticas de Salud. 2020;19. https://doi.org/10.11144/Javeriana.rgps19.prsp

\footnotetext{
a Autora de correspondencia. Correo electrónico: sandra.zuluagas@udea.edu.co
} 


\section{Resumen}

En 1956 se formaron las primeras promotoras de salud en Colombia, para mejorar la salud de las poblaciones de los territorios más marginados. Las promotoras, especialmente mujeres, se convirtieron en protagonistas de la vida comunitaria rural, al jugar un papel que trascendió el quehacer sanitario. El estudio se propuso comprender críticamente el rol social y sanitario de las promotoras de salud en el municipio de Andes, Antioquia, en el periodo 1976-2015. Se realizó un estudio de caso a través de entrevistas y revisión documental; participaron promotoras de amplia trayectoria en ejercicio laboral, funcionarios y líderes comunitarios. La institucionalización de las promotoras irrumpió como la principal alternativa de salud para las comunidades rurales del país. Las transformaciones del sistema de salud han producido el constreñimiento de esta práctica social, aun cuando continúan vigentes. Las promotoras han jugado un papel que trasciende la atención sanitaria, desempeñan un importante rol en la construcción de tejido social y la gestión del cambio sociocultural en la ruralidad colombiana.

Palabras clave: salud rural, promoción de la salud, atención primaria de la salud, promotoras de salud, salud colectiva, participación social, género.

\section{Abstract}

In 1956 the first health promotion agencies were founded in Colombia and were intended to enhance the health condition in the populations in the most alienated territories. The promotion agents, especially women, became leading actors in the rural community when their role transcended the public health endeavors. This study seeks to critically understand both the social and public-health roles of the health promoters in the town of Andes, Antioquia during 1976-2015. A study case was conducted using interviews and document reviews. Female promoters with a wide track record, public servants and community leaders took part in the study. The institutionalization of the promoters emerged as the main health alternative for the rural communities in our country. The transformations in the health system have brought about some pressure on this social practice, even though they still prevail. The promoters have had a role with an impact beyond the public-health care as they perform an important function in the construction of the social fabric and the management for a social cultural change in the Colombian rural life.

Keywords: rural health, health promotion, primary health care, health promotion agencies, collective health, social participation, gender.

\section{Resumo}

Em 1956 formaram-se as primeiras promotoras de saúde na Colômbia, para melhorar a saúde das populações dos territórios mais marginalizados. As promotoras, especialmente mulheres, tornaram-se protagonistas da vida comunitária rural, desempenhando um papel que transcendeu o quefazer sanitário. O estudo propôs compreender criticamente o papel social e sanitário das promotoras de saúde no município de Andes, Antioquia, no período 1976-2015. Foi realizado um estudo de caso por meio de entrevistas e revisão documental; promotoras de vasta experiência no exercício laboral, funcionários e líderes comunitários participaram. A institucionalização das promotoras irrompeu como a principal alternativa de saúde para as comunidades rurais do país. As transformações do sistema de saúde produziram o constrangimento desta prática social, mesmo quando ainda estão em vigor. As promotoras desempenham um papel que transcende a atenção sanitária, muito importante na construção de tecido social e na gestão de mudança sociocultural na ruralidade colombiana.

Palavras-chave: saúde rural, promoção da saúde, atenção primária da saúde, promotoras de saúde, saúde coletiva, participação social, género. 


\section{Introducción}

La Atención Primaria de la Salud (APS) fue planteada en el ámbito internacional a finales de la década de 1970; la declaración de Alma Ata impulsó la institucionalización de esta perspectiva. En su versión comprensiva se entendió como una estrategia para mejorar las condiciones de salud de las poblaciones y la equidad en los resultados sanitarios, a partir de la reorganización del sistema de salud y el reconocimiento de la salud como un derecho humano, mediante el uso de métodos y tecnologías socialmente aceptables, el acercamiento de los servicios de salud a los lugares donde viven y trabajan las personas, la participación social y comunitaria en la gestión de la salud, y la reorientación de los servicios a partir de las necesidades sociales (1)

Uno de los actores clave de la implementación de la estrategia de APS lo constituyen las promotoras de salud, originalmente destinadas al trabajo en las zonas rurales y apartadas del país. Las promotoras rurales de salud surgieron como iniciativa del salubrista Héctor Abad Gómez 1 en la década de 1950 (2), posteriormente en 1976, en el contexto del desarrollo del Sistema Nacional de Salud, a partir de la reglamentación del ejercicio de la enfermería, las promotoras de salud fueron incorporadas como la base del naciente Sistema Nacional de Salud (3).

Originalmente, las promotoras de salud fueron mujeres de origen rural seleccionadas para hacer parte de los equipos de salud. Fueron capacitadas en prácticas para la higiene personal, la educación en salud, el saneamiento ambiental, los primeros auxilios en el hogar, el mejoramiento de vivienda y la vacunación. Se pretendía que mediante la acción educativa y la activación comunitaria se disminuyeran los indicadores de enfermedad y muerte de los territorios rurales $(3,4)$.

Desde la segunda mitad del siglo XX hasta el presente, los sistemas de salud en el mundo han sufrido profundos cambios en sus formas de organización, donde las reformas de mercado han predominado en los sistemas de salud de la región latinoamericana en general, y de manera particular en Colombia en las últimas tres décadas.

A pesar de haber surgido en un contexto socioepidemiológico y político normativo muy diferente del actual, las promotoras de salud continúan siendo reconocidas como un actor protagónico del sistema de salud en el ámbito nacional. No obstante, su relevancia en la gestión de la salud en las comunidades rurales y apartadas del país es incipiente el conocimiento de las transformaciones del contexto social y de los roles de este importante actor de la estrategia de APS. Esta investigación se propuso describir las transformaciones del quehacer de las promotoras de salud en el municipio de Andes, Antioquia en el periodo 1976-2015, por lo que se definió como pregunta orientadora: ¿cuál fue el rol sanitario y social de las promotoras de salud y cómo cambió con las transformaciones de la política de salud en el periodo 1976-2015?

El municipio de Andes está localizado en la subregión Suroeste del departamento de Antioquia, a $117 \mathrm{~km}$ de la ciudad de Medellín. Cuenta con siete corregimientos consolidados, conformados por 63 veredas y por 22 barrios en el área urbana (según la Alcaldía de Andes). Tiene una 
población de 45.814 habitantes, $51 \%$ de sexo masculino y $49 \%$ de sexo femenino, $47 \%$ habitan la zona urbana y 53\% la rural. La principal actividad económica es la agrícola, donde predomina el cultivo de café, en menor escala existen cultivos de plátano, banano, caña de azúcar, yuca y maíz. Otras actividades productivas relevantes son la ganadería, la extracción de materiales de playa y el comercio. El municipio un epicentro regional de negocios y servicios sociales.

\section{Materiales y métodos}

Se realizó una investigación cualitativa entre el segundo semestre de 2015 y el primero de 2016. El recorte temporal de la investigación fue definido en consideración del momento histórico en que las promotoras de salud fueron reconocidas como una ocupación del campo de la salud, en el marco del desarrollo del nuevo Sistema Nacional de Salud definido en Colombia en la década de 1970, y de la institucionalización internacional del discurso de la Atención Primaria de la Salud.

La estrategia metodológica definida fue el estudio de caso, que se consideró pertinente dada la imposibilidad de separar el fenómeno estudiado de su contexto. El caso fue la práctica social de las promotoras de salud del municipio de Andes. La combinación de técnicas en el marco del estudio de caso se realizó con una intencionalidad comprensiva (5).

Se utilizaron dos técnicas para el análisis del caso: la revisión documental y la entrevista, ambas en diferentes momentos del proceso investigativo. Se realizaron 14 entrevistas a promotoras, funcionarios municipales y líderes comunitarios. Las entrevistas se orientaron hacia la identificación de los roles y los cambios históricos; en las promotoras, además, al reconocimiento de las perspectivas de su quehacer y su trayectoria laboral, siguiendo un modelo conversacional y no un intercambio formal de preguntas y respuestas (6).

La revisión documental incluyó fuentes escritas producidas durante el periodo 1976-2015. Se revisaron documentos normativos nacionales y regionales relacionados con la estrategia de APS, así como manuales de formación, lineamientos de promotores de salud del departamento de Antioquia y relatorías de conversatorios relacionados con la implementación de la APS. La revisión documental se orientó a entender las orientaciones técnicas y metodológicas, definidas desde instancias gubernamentales, respecto al quehacer de las promotoras de salud, así como a caracterizar los cambios del contexto político, normativo e institucional.

Para la selección de las promotoras se indagó con un funcionario clave de la Secretaría de Salud municipal, por las promotoras que presentaran una experiencia laboral superior a 20 años, con un ejercicio predominante en zonas rurales, y que estuvieran en ejercicio activo a la fecha. Se identificaron siete promotoras de salud residentes en el municipio de Andes que cumplían con estos criterios, todas ellas fueron formadas entre 1976 y 1993, y continuaban ejerciendo su ocupación en el 2015. Luego de ello, se extendió una invitación a las promotoras para participar de la investigación. De estas, cuatro atendieron el llamado, las tres restantes 
manifestaron no tener interés o presentar alguna situación personal que les impedía participar del estudio. La ejecución inició con la socialización del proyecto a las promotoras y la obtención del consentimiento informado.

Las entrevistas se realizaron en cuatro momentos. El primero fue una entrevista grupal, dirigida a la activación de la memoria (7), para ello se realizó un grupo conversacional con las cuatro promotoras, en el cual se elaboró colectivamente una línea de tiempo, en la que se identificaron los momentos de formación y los hitos de su trayectoria laboral. Como producto de un primer análisis de la entrevista, complementada con la revisión documental, se elaboró una periodización inicial y una definición de categorías preliminares, que fueron utilizadas para orientar las entrevistas individuales posteriores. El segundo momento fue la realización de entrevistas individuales a profundidad con cada una de las promotoras, inicialmente para conversar sobre la historia personal y la trayectoria laboral, en función de las categorías emergentes del primer momento.

El tercer momento fue la realización de entrevistas individuales a funcionarios y líderes comunitarios. Se incluyeron líderes de los lugares donde laboraron las promotoras, que fuesen residentes con más de cinco años de antigüedad en el territorio. Se realizaron tres entrevistas a estos líderes comunitarios, se excluyó un corregimiento por motivos de inseguridad derivada del conflicto armado en la zona. Además, fueron entrevistados tres actores institucionales, profesionales de salud, que se desempeñaron como funcionarios coordinadores de la estrategia de APS en la Gobernación de Antioquia desde inicios de 1980 hasta inicios de la segunda década del 2000 , momento en el que se jubilaron. Estos actores jugaron un papel central en la definición de los criterios técnicos de la implementación de la estrategia y de los procesos de formación de las promotoras durante las tres décadas analizadas. El cuarto momento fue la realización de una segunda ronda de entrevistas individuales con las cuatro promotoras para profundizar en temas de interés identificados en los momentos anteriores.

El proceso de análisis de la información se orientó por las categorías teóricas contexto, roles y significados. El contexto fue entendido como las condiciones particulares del entorno político, normativo e institucional en el cual se realizó la acción de las promotoras; los roles fueron entendidos como las funciones y el papel jugado por las promotoras en relación con la acción sanitaria y social-comunitaria; los significados fueron entendidos como los sentidos e ideas otorgadas por las personas frente a las promotoras y su quehacer sanitario y social, en esta categoría emergió como subcategoría relevante los significados atribuidos a las relaciones de género y el empoderamiento femenino, entendido como las ideas respecto al papel de las promotoras respecto a las relaciones entre las personas en hombres y mujeres y su condición de género.

Finalmente, a partir de la información documental revisada y de las entrevistas realizadas, se hizo una codificación apoyada en una matriz que puso en relación la periodización establecida y las categorías de análisis. Una vez realizado el análisis, se socializaron los resultados con las promotoras, en un último encuentro del proyecto de investigación. 


\section{Resultados}

En Colombia, las promotoras de salud fueron reconocidas legalmente como una ocupación desde 1976; no obstante, su formación fue impulsada decididamente desde instancias gubernamentales del orden nacional y departamental entre 1960 y 1980 (3). La práctica de esta ocupación ha cambiado conforme se ha transformado el contexto político e institucional nacional y territorial.

Las transformaciones en esta trayectoria se pueden leer en tres periodos: un primer momento (1976-1993), reconocido por el impulso dado por la declaración internacional de la APS, en el marco de un Sistema Nacional de Salud, SNS, que poseía unos criterios de organización favorecedores de la APS $(8,9,10,11)$. Un segundo periodo (1994-2005), que corresponde con el cambio del sistema de salud, en el que se implementó el nuevo Sistema General de Seguridad Social en Salud, SGSSS, establecido mediante la Ley 100 de 1993 (12), que redefinió la organización del sistema de salud bajo criterios de mercado y fue reestructurado por una lógica de aseguramiento individual (13), que disminuyó de esta manera la participación del Estado en el manejo de los servicios de salud. Finalmente, un tercer periodo (2006-2015) de retorno a la APS, motivado por la convergencia del movimiento internacional de renovación de la estrategia, la promulgación de nuevas disposiciones normativas de orden nacional y el auge de apuestas políticas de gobiernos locales en el paí s $(4,14,15)$.

\section{El quehacer de las promotoras de salud: un rol socio-sanitario transformado al compás de los cambios de los sistemas de salud}

Finalizando la década de 1950, las promotoras rurales de salud inauguraron una nueva forma de construir la salud en territorios históricamente marginados de la presencia estatal, y donde se expresaba con mayor crudeza el conflicto social derivado de profundas desigualdades sociales. Inicialmente implementado en el municipio de Santo Domingo en el departamento de Antioquia en 1956, bajo la tesis de que las enfermedades y muertes de mayor prevalencia en las zonas rurales eran fácilmente diagnosticables, tratables y evitables, y que no se requería un especialista para ello. Este desafío fue encargado a mujeres que cumplieran con criterios básicos como: un liderazgo socialmente reconocido, el interés manifiesto por trabajar en asuntos comunitarios, habilidades de lectoescritura desarrolladas, y la pertenencia a las propias comunidades donde se desarrollaría la estrategia $(2,3)$. Mujeres que fueron seleccionadas en común acuerdo con las propias comunidades a través de la Acción Comunal, y que realizarían un trabajo voluntario. La implementación de esta iniciativa ha sido reconocida como un hito del desarrollo histórico de la salud pública en Colombia $(4,16)$.

Desde la década de 1960, el entonces Ministerio de Salud Pública había definido seis programas de implementación obligatoria a nivel local, por parte de los centros de salud y la administración de las direcciones departamentales de salud: atención materna e infantil, saneamiento ambiental, control de enfermedades transmisibles, odontología preventiva, estudio y desarrollo de la 
comunidad, y educación para la salud. En todos ellos se entendía que el papel de la promotora de salud era crucial, principalmente en las zonas rurales y con un carácter de voluntariado, por lo cual se produjeron manuales orientadores de la acción y para la formación (17). Inicialmente, los primeros cursos realizados desde el Ministerio de Salud tenían una duración de dos meses intensivos y con dedicación exclusiva, luego se instalaba la promotora en la vereda (3).

Durante las décadas de 1960 y 1970, previo a la institucionalización del discurso de la Atención primaria de la Salud, promovido desde la declaración de Alma Ata, las promotoras de salud jugaron un papel protagónico en el desarrollo del programa materno-infantil impulsado por el Ministerio de Salud (18). Como parte del Sistema Nacional de Salud, el Ministerio de Salud reglamentó el programa de promotoras a través del Decreto 2184 de 1976, que definió unas funciones y una asignación salarial. La promotora de salud fue reconocida como parte del equipo de salud, constituyéndose en la base de la pirámide del Sistema Nacional de Salud. En el departamento de Antioquia, las promotoras se vincularon al Servicio Seccional de Salud departamental en 1978 (3).

Esta reglamentación estuvo influenciada por planteamientos que al poco tiempo fueron incluidos en la Conferencia Mundial sobre Atención Primaria de la Salud realizada en 1978, en la que se planteó que la APS constituye la función central de los sistemas de salud y representa el primer contacto con la atención en salud de los individuos, las familias y la comunidad (19, 20). Los acumulados previos relacionados con la implementación del programa de promotoras rurales junto a otros desarrollos tempranos del Sistema Nacional de Salud hacían parecer que la incorporación de la orientación general de la APS en Colombia sería sencilla (10).

Al finalizar la década de 1970, las promotoras realizaban una amplia variedad de actividades en temas como la participación en la atención prenatal y de la puérpera, en programas prioritarios como el de malaria, tuberculosis y el programa ampliado de inmunizaciones, en la atención de niños y niñas menores de 5 años (crecimiento y desarrollo), en el desarrollo de acciones de prevención de la enfermedad diarreica aguda y la infección respiratoria aguda, en las actividades de planificación familiar, de saneamiento ambiental, en la educación sanitaria y la derivación psicoafectiva $(3,9,21,18)$; en general la promotora aseguraba la remisión oportuna a las instituciones de salud y la aplicación de los tratamientos ordenados por los médicos de las unidades de salud (3). Para ese entonces, el programa nacional de promotoras contaba con 650 en el área rural de 15 departamentos y 76 municipios del país (22).

Durante este período, la cooperación técnica internacional impulsó una gestión de la salud orientada por la APS, a la cual se atribuyeron resultados importantes en la atención de poblaciones prioritarias, principalmente en el área materno-infantil y en el control de enfermedades prioritarias. Gran parte de los proyectos de cooperación se articularon a través de la estrategia de los Sistemas Locales de Salud (Silos), asumidos como el instrumento operativo de la APS (10). El papel de la promotora rural de salud en los Silos se orientó hacia el trabajo comunitario con grupos primarios y a fomentar la creación de grupos organizados en las comunidades (9). 
Durante el desarrollo del Sistema Nacional de Salud se definieron unidades de cobertura, que era la unidad funcional para prestación de servicios básicos de salud en áreas rurales, las cuales eran atendidas por un promotor de salud con una cobertura entre 80 y 120 viviendas en la vereda. Estas unidades pertenecían, a su vez, a una Unidad Primaria de Atención (UPA), que brindaba servicios básicos de atención en salud en zonas rurales, y contaba con una enfermera y una auxiliar de enfermería que coordinaba las acciones de cuatro a ocho promotores. Administrativamente, las UPAS dependían del centro de salud u hospital local más cercano (23).

Entre las décadas de 1970 y 1980, las promotoras se consolidaron como actor clave de la implementación de la Atención Primaria de la Salud. El rol principal definido fue el de constituir primer punto de contacto y enlace entre los servicios de salud y las comunidades (4). La práctica que se fue configurando desplegó una acción situada en el territorio rural que giró en torno a dos grandes ejes, uno en relación con la salud, y otro más ubicado hacia la acción social y comunitaria. En 1981 había 461 promotores en Antioquia; a finales de esa década, el país ya contaba con 4985 promotoras rurales, 781 en Antioquia, y 20 en el municipio de Andes (3, 24).

Un rasgo relevante de las funciones asignadas a la promotora de salud fue la orientación al fomento de la participación de la comunidad en asuntos relacionados con la conservación de la salud y la adecuada utilización de los servicios; la prevención e identificación de riesgos que afectasen la salud; la determinación de prioridades y elaboración de planes de trabajo en las unidades de salud (24).

Una herramienta clave del trabajo de las promotoras de salud la constituyó la identificación comunitaria de los principales problemas de salud y su relación con las condiciones sociales, ambientales, políticas y culturales del territorio. Trabajo realizado a través de estrategias diagnósticas como los mapeos territoriales, los diagnósticos familiares y comunitarios participativos. Con ello, las promotoras lograban un reconocimiento detallado de las particularidades del territorio y de las condiciones familiares:

Íbamos a la comunidad a hacer el diagnóstico comunitario en salud, convocábamos a la comunidad, explicábamos el proceso que íbamos a hacer; construíamos un mapa y el compromiso era reunirnos para complementar el diagnóstico. Íbamos de vivienda en vivienda, con los grupos organizados y formábamos un diagnóstico donde arrojaban todas las necesidades de la vereda, para saber qué trabajar y con eso arrancábamos (Relato promotora de salud).

Las acciones de promoción de la salud, prevención y atención básica de las enfermedades prevalentes se realizaron con una clara orientación familiar y comunitaria, en dicho marco, ejercieron un rol educativo. El despliegue de las acciones sanitarias de carácter asistencial se caracterizó por su amplitud e intención resolutiva, en el que se incluyeron acciones como las enunciadas previamente, además de otras como la atención de partos, la dispensación de medicamentos básicos y la atención de primeros auxilios. 
Las promotoras rurales de salud: una práctica social en extinción.

Estudio de caso en el municipio de Andes, Antioquia, 1976-2015

\begin{abstract}
Nosotras hacíamos los controles a los niños, a las maternas, éramos las parteras —me tocó atender 11 partos - también suturamos heridas superficiales en el campo, también vacunábamos, poníamos todas las vacunas. El trabajo en el campo era muy bueno, las personas le tenían a uno más confianza y cuando eso la situación era más difícil. A las mamás les quedaba difícil venir a la cabecera municipal, entonces las remisiones eran muy poquitas. Es más, en esa época casi que nosotras éramos hasta médicas, porque hasta cargábamos un stock de medicamentos (Relato promotora de salud).
\end{abstract}

También las promotoras jugaron un importante papel en el acercamiento de ofertas institucionales no solamente sectoriales, y el fortalecimiento de la organización comunitaria a través de asambleas y convites dirigidas a la generación de soluciones concretas a las problemáticas comunitarias observadas y a fortalecer la organización comunitaria para la gestión de respuestas en aspectos relacionados con el mejoramiento de vías de acceso, la mitigación de riesgos ambientales, la realización de jornadas de saneamiento para el manejo correcto de basuras y aguas residuales. Las acciones desarrolladas expresaron una clara intención de articulación entre las temáticas de salud, sociales y ambientales, mediadas por la participación de las comunidades:

\begin{abstract}
Algunos logros fueron la instalación de unidades sanitarias, la ampliación de la institución educativa, el mejoramiento de placas polideportivas, la unión de la junta de acción comunal, mejoramiento de vivienda, programas de alfabetización de personas que no sabía escribir, ni leer, hubo nocturna, mejoró la nutrición por las huertas caseras, el mantenimiento comunitario de las carreteras mediante convites (Relato promotora de salud).
\end{abstract}

Al finalizar la década de 1980, una evaluación realizada por el Servicio Seccional de Salud de Antioquia reconocía que, como producto de la ampliación de cobertura basada en las promotoras de salud, propuesta desde el Ministerio de Salud, generó un incremento en las funciones del promotor, que ocasionaba la deserción o la suspensión de actividades por una subestimación de sus tareas; así mismo planteaba la necesidad de acompañar el aumento de promotoras con un correspondiente aumento en el recurso humano dedicado a la supervisión, asesoría y educación continua, reconocía problemas en la capacitación y evaluación de las promotoras, y planteaba la necesidad de flexibilizar los criterios de selección de áreas y promotores para adecuarse a las particularidades socioeconómicas y culturales de las subregiones del departamento $(3,24)$.

A partir de ello se formuló una propuesta de reestructuración del programa de promotores de salud, en el cual se planteó una ampliación del periodo de capacitación a seis meses y la adopción de un enfoque de riesgo, la ocupación de las plazas vacantes tanto del área urbana como rural, la actualización del proceso de capacitación de los promotores indígenas, y la flexibilización de criterios de selección de áreas y promotores. En dicho programa se plantearon como funciones básicas del promotor: a) el fomento de la participación comunitaria para la conservación de la salud y la adecuada utilización de los servicios; b) la promoción de la participación de la comunidad en la detección de riesgos en salud; c) la participación con la comunidad y la unidad de salud en la identificación de factores de riesgo, que permita la definición de prioridades y la elaboración de planes de trabajo; d) la realización de acciones de tratamiento y recuperación de la salud $(3,24)$. Es llamativo, en este plan de reestructuración del programa de promotoras de salud del departamento de Antioquia de 1987, el marcado énfasis en destacar las labores 
sanitarias a la vez que el bajo reconocimiento de la dimensión social y ambiental del quehacer de las promotoras.

Iniciando la década de 1990, se produjeron profundos cambios normativos en el sector salud. Al iniciar la década se sancionaron las Leyes 10 de 1990, que reorganizó el Sistema Nacional de Salud (25), y 60 de 1993, que definió las competencias y recursos de los entes territoriales y de la Nación para la provisión de algunos servicios sociales esenciales (26), con los cuales se impulsó la descentralización y municipalización de la salud, lo que generó desafíos inéditos para los entes territoriales, especialmente por la adopción de una nueva responsabilidad en el nivel municipal, a partir de ese momento responsable por el financiamiento y la conducción del sistema de salud. La descentralización de la salud se vio limitada por la precaria situación económica nacional, y por la baja capacidad de gestión municipal existente en el país. Sin embargo, el cambio más relevante se dio en 1993, con la creación de un nuevo sistema enmarcado en un concepto de seguridad social integral, con la llamada Ley 100 que creó el Sistema General de Seguridad Social en Salud, aún vigente (12).

El nuevo marco político-institucional creado con la Ley 100, aunque fue concebido como una normativa para la organización de la seguridad social, generó un fuerte impacto en el sistema de salud. Con la nueva ley se separó de manera tajante la provisión de los servicios de atención en salud individual, de las acciones colectivas, a las que denominó como de salud pública. Las primeras quedaron supeditadas al esquema de aseguramiento individual a cargo de las nuevas entidades aseguradoras creadas por el sistema, mientras las segundas se mantuvieron como responsabilidad del Estado a través de los entes territoriales y los hospitales públicos. En este lapso, el desarrollo de los Silos y la estrategia de APS se vieron debilitados $(10,27)$.

Con la Ley 100 se instauró una lógica de mercado en la organización y gestión del sistema de salud. A partir de ese momento dichas transformaciones propiciaron un cambio en el rol de las promotoras, en adelante reubicadas hacia el interior de los servicios hospitalarios, desestimulando su labor en el ámbito social y comunitario (4).

La reforma del sistema de salud significó un recorte del rol de las promotoras de salud dentro de su quehacer sanitario, al reducir el alcance de las acciones previamente definidas, en el que fueron suprimidas algunas como la entrega de medicamentos, la atención de partos, la realización de suturas y la aplicación de inyecciones, acciones que se reubicaron a una ejecución exclusiva en los puestos de salud.

También se dio una ampliación del nivel territorial de referencia de la actuación, pasando del nivel de vereda a corregimiento, aumentando con ello el número de familias cubiertas, lo cual limitó las posibilidades de interacción y la intensidad del acompañamiento a las familias. En adelante, la asignación del territorio donde debía trabajar la promotora de salud no consideró la pertenencia o residencia en el mismo: 
Las promotoras rurales de salud: una práctica social en extinción.

Estudio de caso en el municipio de Andes, Antioquia, 1976-2015

Ahí las cosas cambiaron, el promotor ya no era sino de promoción y prevención, mas no de atención, no se iban a hacer atención, ni partos. En el 97 ampliaron el número de veredas a cada promotora, no era un buen trabajo, porque no se podía hacer todo a las familias, porque ya no alcanzaba el tiempo para hacer de todo (Relato promotora de salud).

También con la Ley 100 se reestructuraron las Direcciones Territoriales de Salud. A la vez que se delegó en los municipios la responsabilidad de la asignación de subsidios en salud para la población pobre y el control de la calidad de los servicios de salud, y se llevó a cabo la conversión de los hospitales públicos locales en Empresas Sociales del Estado, ESE, quienes debían garantizar la viabilidad financiera, técnica y administrativa de la institución a través de la venta de servicios (28). Estos cambios, en su conjunto, podrían explicar el desdibujamiento de la estrategia de APS en el sistema de salud del país, que culminó en 1997 con la desvinculación de las promotoras de las instituciones y la eliminación del cargo.

Las transformaciones políticas, normativas e institucionales de la década de 1990 produjeron un deterioro en la situación de la salud pública y de las condiciones de los actores locales para su desarrollo $(20,27,29,11)$. Así mismo, estos cambios significaron una precarización y pérdida de protagonismo de las promotoras, que se acompañó de una percepción generalizada de crisis del sistema de salud, incluida su capacidad de resolución de los problemas, que aún permanece vigente en la segunda década del siglo XXI $(27,30)$ :

Nos desvincularon en 1997, por la Ley 100 se había transformado mucho la salud y que entonces eso iba a depender de otras personas. La Seccional de Salud ya no tenía que ver con nosotras, ahí empezó el tiempo en que contrataba el municipio y el hospital. En esa época nos desmontaron de la vacunación, nos dijeron ustedes no vacunan más, porque eso es atención y ustedes solo hacen prevención, ahí decayó mucho el programa de vacunación (Relato promotora de salud).

En el periodo 1994-2005 no existieron orientaciones explícitas para la implementación de la estrategia de APS en el país y el departamento, por ello el trabajo de las promotoras fue discontinuo y discrecional a las decisiones municipales o de las Empresas Sociales del Estado.

En este periodo tomó forma la segmentación de la atención de la población de acuerdo con el tipo de aseguramiento, según el nuevo arreglo institucional instaurado por el nuevo Sistema General de Seguridad Social en Salud (9). La población fue clasificada por su capacidad de pago: los contribuyentes y sus familias conformaron el régimen contributivo y los regímenes especiales ${ }^{2}$, los ciudadanos pobres y sus familias, que fueran clasificados como tal, recibirían un subsidio estatal y conformaron el régimen subsidiado. En este contexto, la población afiliada al régimen subsidiado fue atendida de manera mayoritaria en los reformados hospitales públicos, devenidos en Empresas Sociales del Estado, y la población afiliada al régimen contributivo fue atendida, mayoritariamente, en hospitales y clínicas privadas.

El nuevo escenario de competencia entre instituciones prestadoras de servicios de salud estuvo marcado por la búsqueda de rentabilidad financiera y la supervivencia de las ESE, lo cual repercutió en el bajo interés de los hospitales públicos por mantener la figura del promotor 
de salud dentro de sus modelos de gestión. En el municipio de Andes, las promotoras fueron contratadas por la Empresa Social del Estado, ESE San Rafael, de manera ocasional. En todos los casos, mediante contratos cortos de una duración no superior a los seis meses.

Además de esto, tuvo lugar la aparición de nuevas disposiciones normativas, relacionadas con la participación comunitaria y el desarrollo de acciones colectivas de prevención de la enfermedad y promoción de la salud a cargo de los entes municipales. Para lo primero, se crearon los Comités de Participación Comunitaria en Salud, Copacos, y para lo segundo se definió el Plan de Atención Básica, PAB (31). En ambos casos, de responsabilidad municipal. Las disposiciones para el desarrollo de acciones grupales de salud pública que determinó la Resolución 4288 de 1996 del Ministerio de Salud se orientaron hacia la educación a nivel grupal, y se eliminó la vacunación domiciliaria que fue reubicada en el nivel hospitalario. A la par de ello, y como parte de la redefinición de responsabilidades en los actores del sistema, también se definió un conjunto de actividades preventivas individuales, relacionadas con la demanda inducida, la protección específica, la detección temprana y la atención de enfermedades de interés en salud pública, establecidas como de obligatorio cumplimiento y de responsabilidad de las aseguradoras (32). Tanto las coberturas de las acciones colectivas, del Plan de Atención Básica, como las preventivas individuales han sido bajas, mientras que la participación se ha caracterizado por su carácter instrumental e institucionalizado $(29,11)$.

Estos cambios terminaron de consolidar la fragmentación de la acción en salud pública y la pérdida de la integralidad de la atención a la enfermedad, al separar las fuentes de financiación y las responsabilidades, de un lado acciones colectivas a cargo de los municipios, y de otro las acciones individuales a cargo de las aseguradoras. En conjunto, estos cambios condujeron a la reducción del quehacer de la promotora, destinada primordialmente a la identificación de población en riesgo y su remisión a los servicios de salud, y circunscrita al alcance de la acción de los debilitados hospitales públicos, a la vez que el papel social-comunitario y ambiental de las promotoras fue anulado.

En este contexto político e institucional, con una mayor autonomía municipal en el manejo de la salud y la presión por la rentabilidad financiera, la vinculación del personal de salud quedó expuesta al vaivén de las cercanías y afiliaciones político-partidarias, lo que produjo discontinuidad en la vinculación de las promotoras de salud y en el desarrollo mismo de la estrategia de APS:

Se juntaron dos cosas: la Ley 100 y la politiquería, por política se vincularon o no a las promotoras, así no supieran lo que iban a hacer, porque si era de la cuerda del alcalde de turno, pues la iban a contratar (Relato promotora de salud).

A la par, en este periodo se conocieron diversas acusaciones sobre el desvío de dineros de la salud a otros campos, atrapados en las ineficiencias de la intermediación y en las redes de corrupción 
territorial. Todo ello fue dando forma a una nueva crisis hospitalaria que demostraba los límites de las políticas implementadas y los efectos del nuevo SGSSS (33).

El tercer periodo, 2006-2015, se caracterizó por una vuelta a la escena de la estrategia de APS, dado por la convergencia de discursos internacionales, de nuevas disposiciones normativas de orden nacional, y especialmente por nuevas apuestas de política de salud de gobiernos departamentales, distritales y municipales.

En Bogotá, en el 2004, en el marco de una experiencia de gobierno alternativo de un partido político no tradicional, se implementó una política local de salud orientada por la APS, que, dado el peso político del gobierno de la capital del país, puso nuevamente sobre la mesa la necesidad de recuperar la APS como estrategia de reorganización del sistema de salud (20).

Con el decreto 3616/2005 (34) se redefinieron los perfiles ocupacionales y de formación de los auxiliares en las áreas de la salud, se eliminó definitivamente el promotor de salud y fue definido un nuevo perfil, denominado técnico en salud pública: "Nos formaron como técnicas de salud pública del Sena, pero eso no cambió nada, seguimos siendo las promotoras de salud con el mismo sueldo y las mismas actividades" (Relato promotora de salud).

En el año 2006, la Gobernación de Antioquia decidió impulsar la iniciativa de APS a través de la estrategia denominada "Promotores y promotoras de vida", justificada en el deterioro de la salud pública y el reconocimiento de las ventajas de la estrategia de APS. Esta iniciativa fue conocida como Pacto por la Salud Pública de Antioquia (4). Su implementación significó la reaparición de las promotoras, cuyo rol se especificó en torno a un conjunto de acciones centradas en el diligenciamiento de un instrumento de caracterización familiar y del entorno, denominado Historia Familiar; además, la remisión a los servicios de salud de individuos clasificados en riesgo, para la realización de las actividades preventivas establecidas en el Plan Obligatorio de Salud y, finalmente, la ejecución de acciones de educación en salud, orientadas especialmente a informar sobre estilos de vida saludable:

En ese tiempo se hacía inducción a la demanda según las necesidades de atención de acuerdo con la edad de las personas, hacíamos la educación, búsqueda de susceptibles y los inasistentes, para realizar principalmente la remisión al hospital. Y solo era vaya y hágale firmar, no hay una preocupación por lo que se hace, solo es cantidad y no calidad (Relato promotora de salud).

Para este periodo, las promotoras realizaron su trabajo al interior de un equipo de profesionales de salud pública que realizaban acciones del renovado PAB, ahora denominado Plan de Intervenciones Colectivas, PIC. La articulación de las acciones entre profesionales y las promotoras fue débil, dadas las complejas y elevadas demandas de las personas y familias, la presión por las coberturas y el limitado tamaño del equipo. La labor de identificación de personas en riesgo y la remisión a los servicios de salud se enfrentó a dificultades para el acceso a los servicios de salud, en la medida en que estaba mediado por la situación del aseguramiento en 
salud de cada persona, más las dificultades geográficas y económicas que supone el traslado hacia los centros de salud de las personas que habitan los territorios rurales.

En el ámbito internacional en 2007, la Organización Panamericana de la Salud promovió la renovación de la APS en las Américas (1), y la Organización Mundial de la Salud presentó en 2008 el informe sobre la salud en el mundo, denominado La APS Más Necesaria que Nunca (35), lo que impulsó nuevamente la APS en las agendas políticas nacionales. La estrategia de APS había vuelto a la escena.

En 2007 se expidió la Ley 1122 de 2007 para establecer ajustes al SGSSS. Se determinó definir un Plan Nacional de Salud Pública, PNSP, y luego de este se inició un proceso de construcción de los lineamientos técnicos para la implementación del plan en el ámbito territorial, este proceso permitió la formulación de los planes de salud territoriales (PST), aún en vigencia, y que favoreció un reposicionamiento de la salud pública y de las actividades preventivas en el país (36).

En 2010, la Dirección Seccional de Salud de Antioquia, presentó los lineamientos para la promoción de la salud en el departamento, recogiendo el acumulado de aprendizajes que iniciaron desde el 2006 (4).

Por su parte, en 2011, luego de varios años de acumulados de apuestas departamentales, distritales y municipales por la reorientación de los sistemas locales de salud a partir de la APS, el entonces Ministerio de la Protección Social cedió a la creciente presión, y por iniciativa gubernamental fue presentada y sancionada la Ley 1438 (15), que introdujo la APS en el Sistema General de Seguridad Social en Salud después de casi dos décadas de iniciada la Ley 100.

La nueva normativa nacional señaló la relevancia de las promotoras de salud en los llamados equipos básicos de salud. A pesar de la acogida que tuvieron dichas disposiciones en el ambiente político sectorial e institucional, la precitada norma no tuvo desarrollo reglamentario, ni fueron asignados recursos para impulsar el trabajo de los equipos básicos de salud, con lo cual la implementación de la estrategia de APS y el trabajo de las promotoras fue discrecional a las decisiones de las autoridades municipales.

Por iniciativa propia, y al margen de consideraciones laborales o contractuales, las promotoras mantuvieron un trabajo de gestión comunitaria, orientado a dinamizar grupos de tercera edad, a la gestión de temas ambientales (huertas, reciclaje, saneamiento) y de educación sanitaria, que en ocasiones está respaldado por su liderazgo en espacios como las Juntas de Acción Comunal. 
Las promotoras rurales de salud: una práctica social en extinción.

Estudio de caso en el municipio de Andes, Antioquia, 1976-2015

\section{Sobre los significados atribuidos y las relaciones de género}

En el proceso de reconstrucción histórica de la práctica de las promotoras de salud en Andes, emergieron significados atribuidos a su quehacer en diferentes ámbitos de la acción y de las relaciones sociales; construidos como producto de una interacción de muchos años entre las personas de las comunidades, los actores institucionales y una identidad asumida por ellas mismas.

Revisar la experiencia histórica de las promotoras de salud permite una reflexión en torno al papel de las mujeres y a las relaciones de género, particularmente en el campo de la salud y en lo que atañe a la construcción de procesos participativos, las mujeres han ocupado un rol destacado, especialmente en los espacios locales, donde se han desenvuelto y proyectado sus papeles, habilidades y luchas. Además, al menos en el campo de la salud, las mujeres constituyen la principal fuerza de trabajo, especialmente en el primer nivel de atención (36).

De hecho, la visibilización de las mujeres y la transformación de su condición, tanto sociológica como subjetiva, obedecen - desde la segunda mitad del siglo pasado en casi todo el mundo occidental y específicamente desde la década del sesenta en Colombia - a sus propias luchas por los derechos de ciudadanía, o sea por una democracia real que las incluya a las demandas por lograr equidad en todos los espacios de la vida, pero también a grandes coyunturas ideológicas, sociológicas, económicas y políticas del país en general (37).

Teniendo como referencia el escenario comunitario como el principal campo de acción de las promotoras de salud, los significados identificados aluden a ideas respecto al empoderamiento femenino, al papel de enlace o puente de comunicación entre la institucionalidad y las comunidades, a la gestión del cambio, así como a la protección y cuidado del tejido social.

Un primer significado identificado se relaciona con la acción de la promotora en salud como una práctica de mujeres empoderadas, que refleja el posicionamiento femenino en el cuidado comunitario de la salud, ya no solo en un ámbito doméstico, sino en un contexto sociocultural de predominante control masculino sobre la acción en la esfera de lo público y en las decisiones de las mujeres:

Yo era una persona muy tímida, de la casa no salía, pero me gustaba enseñar el catecismo, estar aquí y allá... yo no había terminado el bachillerato porque la situación económica de la familia no daba... y me eligieron para ser promotora, pero por lo que mi papá era de los que decía: "no se me mueve si no está acompañada, que no sale, de aquí no te movés, la mujer no está sino para la casa". Tuvo que ir la jefe a mi casa a decirle a mi papá que por qué no me dejaba ir, entonces, cuando llegó ella y me dijo vos que pensás, ¿sí vas a ir? Yo con mucha pena y restringida por allá detrás de una puerta, le dije dígale a mi papá que lo que él diga. Y yo con esas ganas, pero él era el que tomaba mi decisión. Pero entonces ya le dio pena a mi papá y dijo: "si usted se quiere ir, pues vaya". Ahí la jefe me dijo "hágale, que usted tiene capacidad" (Relato promotora de salud).

Para mí es primero mi comunidad, mi esposo me dice "usted es oscuridad en la casa y es claridad en la calle, usted alumbra es para afuera, usted para adentro no alumbra en la casa". Se llegan épocas en que le puede dedicar tiempo al esposo y a los hijos, pero otras no (Relato promotora de salud). 
El constituirse como promotoras de salud implicó una confrontación contra una estructura social patriarcal predominante, cuestión que se mantiene a lo largo de su trayectoria, y que resignificó la identidad de este nuevo sujeto social; un desafío de enorme complejidad en tanto la historia patriarcal construida a lo largo de los siglos, configuró una relación de la mujer afuera de los circuitos del poder, del saber y del placer, siempre bajo la tutela de un hombre, padre, hermano mayor o marido y dotada de un cuerpo disciplinado y al servicio de otros (37).

Otro significado asociado con el hacer de la promotora fue el de constituir un puente o enlace entre la institucionalidad y la comunidad. Desde el momento de institucionalización de las promotoras de salud, fueron consideradas primer punto de contacto y el enlace entre las comunidades y las instituciones, para mediar el desarrollo de las acciones de salud. Ellas realizaron gestiones para superar barreras de acceso y propiciar la cohesión de la comunidad; desde el inicio fueron la única alternativa de salud en la zona rural, acercaron los servicios y brindaron información en el entorno comunitario, fueron quienes personificaron el servicio a la comunidad, además de ser la voz que interpretaba la situación de salud a los actores institucionales.

Como agentes de salud, realizaron acciones para la prevención de enfermedades, basadas en diagnósticos familiares y comunitarios, con las que desplegaron acciones prácticas de gran utilidad para enfrentar, manejar y resolver situaciones de enfermedad, haciendo uso de tecnologías simples para el manejo en casa como la rehidratación oral, el manejo de fiebre, la realización de pequeñas suturas, entre otras. Ello las convirtió en importante referente comunitario.

La mayoría de las familias tienen credibilidad en uno porque se les explica el porqué de las cosas, siempre hemos sido el apoyo de ellos, que la cita y esto, además siempre le hemos hablado con la verdad... confían en nosotros (Relato promotora de salud).

Las promotoras de salud se constituyeron en actores sociales clave que aportaron en la construcción de nuevas oportunidades para sus comunidades. Fungieron como mediadoras entre las instituciones de salud, para poner al alcance de la población y traducir el lenguaje técnico especializado de la salud, a la vez que generaron y promovieron acciones orientadas al cambio social de las condiciones materiales de vida de las comunidades.

Un tercer significado fue el reconocimiento de las promotoras como gestoras del cambio social. El quehacer de las promotoras de salud trascendió el rol sanitario para dirigirse también hacia el fortalecimiento de la organización y la participación comunitaria, lo cual abrió la posibilidad de pensar la salud desde dimensiones más amplias del quehacer sanitario, fuertemente definido desde el conocimiento biomédico dominante. 
La construcción colectiva de mapeos de necesidades permitió la identificación y lectura del territorio desde sus potencialidades y asuntos críticos, que posteriormente se desplegó en acciones colectivas y comunitarias para resolver las problemáticas desde las propias capacidades de las personas y del territorio; así mismo, adelantaron gestiones intersectoriales tendientes a solucionar situaciones problemáticas previamente identificadas.

En el papel como agente de cambio social, se desplegaron acciones micropolíticas en la vida cotidiana y la organización social de las comunidades, con lo cual se aportó en la cohesión social en grupos históricamente marginalizados (38).

También las promotoras trascendieron hacia un rol político como líderes comunitarias reconocidas por la labor en la gestión de transformaciones sociales y culturales, como las de construcción de infraestructura, mejoramiento de viviendas, aprovechamiento y cuidado de recursos naturales, la promoción del cambio en pautas culturales y la gestión del desarrollo social:

Hacíamos la evaluación de los diagnósticos en asamblea y se veía lo que se mejoraba y faltaba por mejorar para acordar la voluntad de las personas y gestionar los recursos y todo lo que se necesitara en la comunidad (Relato promotora de salud).

Este rol apuntaló una construcción de la política en las poblaciones marginalizadas en términos de la construcción de la consciencia del nosotros a partir de la identificación de problemas compartidos y la construcción de intereses comunes (39).

Las promotoras de salud se convirtieron en personajes de notable relevancia para las comunidades, puesto que tenían un reconocimiento como agentes políticos del territorio. En torno a ellas se congregó la población y se organizaron diversas actividades comunitarias. Si alguien quería realizar una actividad en la vereda, se buscaba a la promotora de salud, lo que incluso desencadenó rivalidades con actores políticos locales tradicionales como los presidentes de las Juntas de Acción Comunal:

Eran muy importantes en la comunidad, nadie iba a la comunidad sin que la promotora supiera, hasta el político la buscaba porque era la que más congregaba población. Ella era la que convocaba, reunía a la gente. La promotora era reconocida como el personaje más importante de la vereda, que incluso creó problemas con los presidentes de acción comunal porque decían “¿por qué no me pregunto a mí?” (Entrevista actor institucional).

"Las promotoras llegaron a tener un papel de reconocimiento público y político, y para los hospitales era un recurso que les facilitaba las acciones a nivel comunitario" (Entrevista actor institucional). Tradicionalmente los liderazgos comunitarios y las Juntas de Acción Comunal fueron asumidos por hombres, generalmente, un asunto naturalizado por premisas culturales patriarcales que planteaban el espacio doméstico y privado como propio de la mujer, mientras que el espacio público y político como propio del hombre. Sin embargo, la práctica de las promotoras de salud, relacionada con la realización de diagnósticos participativos y la 
gestión para el mejoramiento de condiciones del entorno, promovió el empoderamiento y posicionamiento de estas mujeres en asuntos públicos y políticos relacionados con la gestión del desarrollo social de las comunidades.

Un último significado se relacionó con el rol de como protectoras del tejido social, dado su trabajo con grupos organizados de la comunidad y el despliegue de acciones de fortalecimiento organizativo. Además, fueron reconocidas como mujeres protectoras y acompañantes de poblaciones vulnerables, como adultos mayores, niños y personas en condición de discapacidad. $\mathrm{Su}$ condición de género fue un facilitador para la realización de acciones que implicaron una disposición sensible al servicio de otros y a construir relaciones de confianza, asociadas con el cuidado de la salud y la vida, que se actúan a través de la agencia y subjetividad (40):

Las mujeres somos más comprensivas, más dedicadas con más entrega en lo que hacemos, los hombres son más duritos, no son personas que se ponen en el lugar del otro, no son tan sensibles como las mujeres (Relato promotora de salud).

Estas acciones de cuidado y protección a grupos, que muchas veces llegaban al espacio íntimo de los hogares, supuso una predilección de la población por la labor ejercida por promotoras mujeres, aunque también implicó la resistencia de sus esposos por la desconfianza que les generaba las visitas a los espacios familiares. En algunos casos, las comunidades rechazaron o no aceptaron la asignación de los pocos promotores hombres que realizan tal labor.

Un trabajo importante en el rol de protección y cuidado del tejido social está sustentado en la acción educativa que realizaron las promotoras de salud, reconocidas como educadoras de la comunidad. Además, como multiplicadoras del saber popular, en zonas donde prevalecen los saberes tradicionales asociados con el uso de los recursos naturales de los que disponen.

Su ejercicio de liderazgo estimuló la creación de redes sociales al interior de su propia comunidad para trabajar en equipo en la búsqueda de problemas de la población, de esta manera la promotora se percibe como pieza fundamental para el desarrollo de su propia comunidad (9).

El reconocimiento social a las promotoras de salud quedó fijado en las comunidades, en la medida en que las promotoras continuaron en el territorio. Sin importar los ajustes normativos, las transiciones laborales y los cambios en el perfil ocupacional, tanto comunidades como funcionarios de las instituciones nombran a estas mujeres como promotoras, aunque el perfil ya no existe formalmente en las disposiciones normativas del país.

Independientemente de la situación laboral, ellas siguieron realizando una labor de acercamiento de los servicios de salud y otros servicios sociales en el territorio. Continuaron gestionando citas en los servicios de salud, tramitando solicitudes ante la Alcaldía Municipal, tendientes a resolver 
problemas de su corregimiento o vereda, esto en la medida que mantuvieron un reconocimiento por parte de las instituciones del municipio:

No voy a dejar de ser promotora, yo creo va a ser de por vida, ¡estoy segura de que será de por vida!, porque así no esté empleada, devengando un sueldo, yo sigo con mi gente. A mí me necesitan es allá donde tengo el postrado en cama, tengo la persona discapacitada que voy a hablar con ella, que lo ayudo a bañar, que la hago reír, ese es el que me necesita, la persona que no puede salir a tomarse la presión. ¡Yo tengo tanto que hacer! (Relato promotora de salud).

\section{Discusión y conclusiones}

Al analizar la trayectoria histórica de las promotoras de salud, se puede concluir que la amplitud del rol que desempeñaron y su visible presencia en el espacio comunitario las convirtieron en un actor de gran relevancia en el equipo de salud y principal soporte del desarrollo de la estrategia de APS y de la salud pública, sujeto al alcance que le permitió la política de salud en cada momento histórico. Este quehacer híbrido, social y sanitario, abrió una posibilidad de resignificación de la salud, no solo en el sentido de una realidad que demanda una respuesta que trasciende las instituciones hospitalarias, sino también en el reconocimiento de esta como proceso social y condición para el desarrollo de las personas y comunidades.

No obstante, la precariedad de las condiciones laborales, el cambio de perfil ocupacional, la discontinuidad de los programas y la debilidad de las políticas sectoriales, las promotoras de salud continúan vigentes; siguen teniendo un rol protagónico en la gestión de la salud en los territorios rurales, que aún carecen de la infraestructura y de programas continuos para promover la salud y prevenir enfermedades completamente evitables en los albores del siglo XXI. Estas mujeres continúan siendo reconocidas por la comunidad como sus promotoras de salud, a pesar de no tener vinculación laboral, o de que haya llegado una nueva promotora, son a ellas a quien acuden. Sigue siendo común que los profesionales y auxiliares del hospital se apoyan en ellas para abrir espacios familiares y comunitarios.

Desde su planteamiento inicial, el programa de promotoras rurales siempre estuvo soportado en un reconocimiento de la realidad colombiana, tendiente a acercar a las familias, sobre todo de las zonas rurales, los servicios de atención sanitaria que eran inaccesibles para un gran porcentaje de la población $(2,3,22)$. Es por ello que los conocimientos y habilidades de las promotoras rurales eran proporcionales a la distancia que se encontrara su territorio de un centro de salud, ya que en una zona muy apartada debía tener una capacidad resolutiva mucho más amplia (18).

Las promotoras desplegaron unas prácticas en salud, en las que además de la propia acción sanitaria se movilizaron sus dimensiones social y política, aportando con ello a una mejor comprensión de los fenómenos sociales de cada comunidad, promoviendo un entendimiento de la salud como proceso y producto social, en tanto determinada por las relaciones sociales, políticas, económicas y culturales de los grupos humanos $(41,42)$. 
El quehacer de las promotoras de salud se afirmó como una práctica social que implicó el reconocimiento particular de una realidad social, histórica y territorial, constituyéndose como actores sociales con una presencia preocupada y activa en el mundo. Las promotoras de salud desplegaron acciones que determinaron y produjeron lo social, en medio de esta relación se configuró "el campo de la salud" como un espacio de interacción de los diferentes actores individuales y colectivos que, a través de acciones e intereses en juego, definieron posiciones sociales para consolidar acumulaciones de experiencias, conocimientos, prestigio y significaciones $(43,44)$.

Las promotoras de salud asumieron un rol de puente y enlace entre las instituciones de salud y los espacios sociales y comunitarios, que tensionó las relaciones de poder entre actores institucionales, como médicos y enfermeras poseedores del prestigio y reconocimiento, en un campo social de poder $(45,43)$, en el que son hegemónicas la institución hospitalaria y el saber biomédico.

La trayectoria de estas mujeres en actividades colectivas y en sus relaciones con las instituciones políticas, tanto formales como informales, desempeñó un rol relevante la construcción de posiciones valorativas sobre las tareas de las propias promotoras de salud (36). En contraste con las relaciones construidas y producidas en el espacio comunitario, lugar donde las promotoras de salud consolidaron vínculos y aparecieron como protagonistas del campo de la salud al poner en juego prácticas en salud contextualizadas y acordes con los intereses de la comunidad, realizadas en los espacios de vida cotidiana, donde se entrecruza el saber popular con los conocimientos técnicos adquiridos, lo cual les permitió ganar legitimidad y credibilidad por los diferentes actores sociales, y otorgó prestigio a su rol como promotoras de salud.

El surgimiento de las promotoras de salud se dio en un contexto de contradicción, dada la influencia predominante de un patrón cultural patriarcal, reflejado en la dependencia económica de la mujer y la baja autonomía para la toma de decisiones, siempre mediadas por la pareja o padre, además del escaso acceso a la educación. En este caso, el empoderamiento de la mujer estuvo acompañado de la participación en la vida social, con lo cual las promotoras de salud se constituyeron en agente de ruptura y cambio en las prácticas tradicionales de sumisión. El empoderamiento en la mujer permite el desarrollo de una participación social activa que le lleva a experimentar un desarrollo positivo de su autoconcepto, en términos de atributos como competencia, confianza en sí misma, persistencia, empatía y coraje (46).

La práctica de las promotoras de salud visibilizó a la mujer en los espacios públicos, al desarrollar capacidades para construir nuevas miradas del mundo y salir del silencio, aportando en el ejercicio político con alternativas renovadas y de transformación desde lo cotidiano (37). Con ello, incidieron en la reorganización de espacios de poder en la cotidianidad de la ruralidad, en el nivel micro, a través de procesos de organización y gestión comunitaria de la salud. 
En un contexto patriarcal, el quehacer de las promotoras propició el despliegue de un rol de cuidado familiar que trascendió del espacio doméstico hacia el entorno comunitario, lo cual propició un reconocimiento de las mujeres en el espacio colectivo, facilitó el desempeño de nuevos roles y la apropiación de espacios asignados tradicionalmente a los hombres, promoviendo procesos de liderazgo y movilización social. Este reconocimiento público generó tensiones con los líderes comunitarios tradicionales, hombres en su mayoría, denotando la dialéctica entre agente y estructura, al permitir evidenciar la transformación de estructuras sociales a través de unas prácticas en constante transformación (45).

La práctica social de las promotoras de salud se enfocó en el cuidado individual y grupal, así como en el fortalecimiento comunitario a través del despliegue de las capacidades colectivas y la construcción de lazos de solidaridad. Esto aportó a la comprensión de la vida y su cuidado, al actuar en la cotidianidad de la vida social a través de disposiciones que adquirieron a través de la experiencia que las consolidó como agentes de cambio. Liderazgo y empoderamiento que impulsó cambios culturales en los imaginarios sociales, especialmente acerca de la relación de la mujer con el poder desafiantes del patriarcado (46).

Se pueden identificar seis disposiciones principales (36) que soportan el quehacer de las promotoras de salud en el caso analizado: a) la disposición al aprendizaje y el rescate del saber local, como la posibilidad de acceder a nuevos conocimientos — para comprender una realidad situada - que podían ser transmitidos a la comunidad; b) la disposición de comunicación, en tanto la visita familiar constituyó un acto comunicativo en sí, para transmitir, enseñar y reproducir conocimiento y saber; c) la disposición al acompañamiento, en la medida en que estaban dispuestas a recibir las solicitudes o quejas de los individuos, familias o comunidad frente al dolor, sufrimiento, las inequidades, y acompañar el proceso de resolución de situaciones problemáticas; d) la disposición a movilizar la participación y organización, como esa posibilidad de mantener la vida colectiva y la organización social; e) la disposición de conocimiento e identidad territorial, en tanto hacían parte del territorio, y la pertenencia al mismo les permitió aportar a la consolidación de una identidad propia; f) la disposición del reconocimiento institucional, en cuanto conocían las instituciones municipales, lo que permitió la gestión de respuestas a las demandas sociales.

Estas disposiciones permiten identificar cómo fue incorporado un sistema de estructuras cognitivas y motivacionales en la cotidianidad de las promotoras, construido por las experiencias históricas acumuladas que fueron interiorizadas como habitus $(43,47)$, que asegura la presencia de determinados esquemas de percepción, de pensamiento y de acción que tienden a garantizar la conformidad de las prácticas y su constancia a través del tiempo (35).

Esta práctica social se encuentra en franco riesgo de desaparecer, producto de las transformaciones en los roles de las promotoras y la desaparición del criterio de pertenencia y permanencia en los territorios, a la vez que por los cambios político-normativos recientes (48), orientados por una visión mercantilizada de la salud, que fortalecen los procesos de orden 
asistencial-biomédico en detrimento de los procesos sociales y comunitarios en la gestión de la salud.

\section{Agradecimientos}

A las promotoras de salud del municipio de Andes, a la enfermera epidemióloga María Eugenia Ramírez, quien coordinó la estrategia de Atención Primaria de la Salud en el municipio de Andes durante 2013-2015; a Sara Cano por su apoyo en revisión de la versión final del manuscrito; a los profesores Gonzalo Jaramillo y Marcela Gómez de la Universidad de Antioquia por su retroalimentación al texto; a Ana María Zuleta, Secretaria de Salud y Bienestar Social del municipio de Andes, por facilitar el desarrollo de la investigación.

\section{Referencias}

1. Organización Panamericana de la Salud/Organización Mundial de la Salud. La Renovación de la Atención Primaria de Salud en las Américas [Internet]. Washington: Organización Panamericana de la Salud; 2007 [revisión 2016 nov. 6]. p. 48. Disponible en: http://new.paho.org/hq/dmdocuments/2010/Reno vacion_Atencion_Primaria_Salud_Americas-OPS.pdf

2. Abad-Gómez H. Anteproyecto de curso para promotores de salubridad rural. Medellín: Universidad de Antioquia; 1959.

3. Cataño L, Chávez BM. Evolución histórica de los promotores de salud en el Servicio Seccional de Salud de Antioquia. Medellín: Servicio Seccional de Salud de Antioquia; 1988.

4. Dirección Seccional de Salud y Protección social de Antioquia. Lineamientos para la promoción de la salud con sentido humano en Antioquia [Internet]. 1. a edición. Medellín: Universidad de Antioquia; 2010. 596 p. Disponible en: https://www.dssa.gov.co/index.php/descargas/733-libro-lineamientos-p romocion-salud/file

5. Yin R. Investigación sobre estudio de casos. Diseño y Métodos. 2. ${ }^{a}$ ed. Nueva York: Sage publications; 2003.

6. Taylor SJ, Bogdan R. Introducción a los métodos cualitativos de investigación: la búsqueda de significados. Barcelona: Paidós; 1992. 344 p.

7. Cendales L, Torres A. La sistematización como experiencia investigativa y formativa [Internet]. Bogotá; 2013 [revisión 2016 nov. 5]. Disponible en: http://www.cepalforja.org/sistem/documentos/lola_cen dales-alfonso_torres-la_sistematizacion_como_experiencia_investigativa_y_formativa.pdf

8. Colombia. Ministerio de Salud Pública. Decreto 350 de 1975, por el cual se determina la organización y funcionamiento de los servicios seccionales de salud y de las unidades regionales. Diario Oficial, 34288 ( abr. 3). Bogotá: Ministerio de Salud Pública; 1975.

9. Castrillón-Montoya JM. Participación comunitaria en promotores de salud, Guarne 1998-2000 [Trabajo de investigación Maestría en Salud Pública]. [Medellín]: Universidad de Antioquia; 2000. 
10. Organización Panamericana de la Salud. La OPS y el Estado colombiano: Cien años de historia 1902-2002. [Internet]. 2002 [revisión 2019 sep. 12]. Disponible en: https://www.paho.org/col/index.php?option=com_docman\&view=download\&category_slug $=$ p ublicaciones-ops-oms-colombia\&alias=61-la-ops-y-el-estado-colombiano-cien-anos-de-historia\&It emid $=688$

11. Gómez A, Eslava JC, Camacho S. Aportes al mejoramiento de la gestión de la salud pública en Colombia. Bogotá: Editorial Kimpres Ltda; 2012.

12. Colombia. Congreso de la República. Ley 100 de 1993, por la cual se crea el sistema de seguridad social integral y se dictan otras disposiciones. Diario Oficial, 41148 (dic. 23). Bogotá: Congreso de la República; 1993.

13. Álvarez MH. El derecho a la salud en Colombia: obstáculos estructurales para su realización. Rev. Salud Pública. 2000;2(2):121-144.

14. Secretaría Distrital de Salud de Bogotá. Atención primaria en salud: un camino hacia la equidad. Bogotá: Corporación para la Salud Popular Grupo Guillermo Fergusson; 2012.

15. Colombia. Congreso de la República. Ley 1438 de 2011, por medio de la cual se reforma el Sistema General de Seguridad Social en Salud y se dictan otras disposiciones. Diario Oficial, 47957 (ene. 18). Bogotá: Congreso de la República, 2011.

16. Apráez G. Evaluación de la atención primaria de la salud en Colombia como política de salud 1939-2008. [Tesis Doctorado en Salud Pública]. [Bogotá]: Universidad Nacional de Colombia; 2010.

17. Colombia. Ministerio de Salud Pública. Manual de la promotora rural de salud. Bogotá: Ministerio de Salud Pública; 1965.

18. Ministerio de Salud. Encuentro Nacional de Promotoras de Salud. Bogotá: Trazo Ltda; 1985.

19. Organización Mundial de la Salud. Declaración de Alma-Ata. En: Conferencia Internacional sobre Atención Primaria de Salud. Washington: Organización Mundial de la Salud; 1978 [revisión 2016 nov. 6]. p. 3. Disponible en: http://www.paho.org/hq/index.php?option=com_docman\&task=doc_vi ew\&gid=19004\&Itemid=270

20. Vega Romero R, Naydú AR, Mosquera PA, Restrepo O. Atención Primaria Integral de Salud. Estrategia para la transformación del sistema de salud y el logro de la equidad en salud. Bogotá: Alcaldía Mayor de Bogotá/Secretaría Distrital de Salud; 2009. 314 p.

21. Servicio Seccional de Salud de Antioquia. Promotor de salud - primer nivel de contacto entre la comunidad y el sistema de prestación de servicios de salud. Medellín: Servicio Seccional de Salud de Antioquia; 1988.

22. Ruiz Marín LA. La medicina social de Héctor Abad Gómez 1944-1964 [Trabajo de investigación Maestría en Historia]. [Medellín]: Universidad de Antioquia; 2015.

23. Servicio Seccional de Salud de Antioquia. Instrucción programada para promotores de salud, Módulos 1 al 20. Medellín: Servicio Seccional de Salud de Antioquia; 1989.

24. Servicio Seccional de Salud de Antioquia. Promotor de Salud. Medellín: Servicio Seccional de Salud de Antioquia; 1988. 
25. Colombia. Congreso de la República. Ley 10 de 1990, por el cual se reorganiza el Sistema Nacional de Salud. Diario Oficial, 39137 (ene. 10). Bogotá: Congreso de la República; 1990.

26. Colombia. Congreso de la República. Ley 60 de 1990, por el cual se dictan normas orgánicas sobre la distribución de competencias y recursos de los entes territoriales y la Nación. Diario Oficial, 23182 (ago. 12). Bogotá: Congreso de la República; 1990.

27. Franco-Agudelo S. Para que la Salud sea Pública: algunas lecciones de la reforma de salud y seguridad social en Colombia. Rev Gerenc Polit Salud. 2003;2(4):58-69.

28. Galán-Morera R. Reorganización del Sistema Nacional de Salud, una gran transformación Ley 10 de 1990. Rev Esc Adm Negocios. 1990;10:13-18.

29. Molina-Marín G, Ramírez-Gómez A, Ruiz-Gutiérrez AM. Tensiones en las decisiones en salud pública en el sistema de salud colombiano: el bien común en confrontación con los intereses y prácticas particulares. Medellín: Universidad de Antioquia; 2013.

30. Yepes F. Luces y sombras de la reforma de la salud en Colombia. Ley 100 de 1993. Rev Gerenc Polit Salud. 2010; 9(18):118-123.

31. Colombia. Ministerio de Salud. Resolución 4288 de 1996, por la cual se define el Plan de Atención Básica (PAB) del Sistema General de Seguridad Social en Salud (SGSSS). Bogotá: Ministerio de Salud; 1990.

32. Colombia. Ministerio de Salud. Resolución 412 de 2000, por la cual se establecen las actividades, procedimientos e intervenciones de demanda inducida y obligatorio cumplimiento y se adoptan las normas técnicas y guías de atención para el desarrollo de las acciones de protección específica y detección temprana y la atención de enfermedades de interés en salud pública. Bogotá: Ministerio de Salud; 2000.

33. Vega-Vargas M, Eslava-Castañeda JC, Arrubla-Sánchez D, Hernández-Álvarez M. La reforma sanitaria en la Colombia de finales del siglo XX: aproximación histórica desde el análisis sociopolítico. Rev Gerenc Polit Salud. 2012;11(23):58-84.

34. Colombia. Ministerio de la Protección Social. Decreto 3616 de 2005, por medio del cual se establecen las denominaciones de los auxiliares en las áreas de la salud, se adoptan sus perfiles ocupacionales y de formación, los requisitos básicos de calidad de sus programas. Diario oficial, 46060 (oct. 10). Bogotá: Ministerio de la Protección Social; 2005.

35. Organización Mundial de la Salud. Informe sobre la salud en el mundo 2008: La atención primaria de salud, más necesaria que nunca. Ginebra: Organización Mundial de la Salud; 2008.

36. Pagnamento L. El trabajo de las Promotoras de salud municipales y la participación social. Análisis de caso. En: VII Jornadas de Sociología de la Universidad Nacional de La Plata "Argentina en el escenario latinoamericano actual: debates desde las ciencias sociales". [Internet]. La Plata: Universidad Nacional; 2012 [revisión 2019 sep. 15]. Disponible en http://jornadassociologia.fahce. unlp.edu.ar/vii-jornadas-2012/actas/Pagnamento.pdf 
Las promotoras rurales de salud: una práctica social en extinción.

Estudio de caso en el municipio de Andes, Antioquia, 1976-2015

37. Thomas FA. Nueva mujer, nuevo hombre. En: Antología del pensamiento crítico colombiano contemporáneo [Internet]. Buenos Aires: Clacso; 2015. p. 523. Disponible en: http://biblioteca.clac so.edu.ar/clacso/se/20151023041701/AntologiaColombia.pdf

38. Gledhill J. El poder y sus disfraces: perspectivas antropológicas de la política. Barcelona: Bellaterra; 2000.

39. Heller A. Sociología de la vida cotidiana. Barcelona: Península; 1977.

40. Santillán L. La crianza y educación infantil como cuestión social, política y cotidiana: una etnografía en barrios populares del Gran Buenos Aires. Anthropologica. 2009; 27(27):47-74. Disponible en: ht tp://www.scielo.org.pe/scielo.php?script=sci_arttext\&pid=S0254-92122009000100004

41. Quevedo E, Hernández M, Cortés C, Eslava JC. Un modelo para armar: Una propuesta metodológica para abordar el estudio comparativo de la historia de la salud pública, de las profesiones de la salud y de sus relaciones de doble vía con la sociedad. Rev Cienc Salud [Internet]. 2013;11(3):295-321. Disponible en: http://revistas.urosario.edu.co/index.php/revsalud/article/view/2789

42. Granda E. Veinte años. Balance y perspectivas de la medicina social. En: La Salud y la Vida, volumen 1. Quito: Ministerio de Salud Pública del Ecuador; 2009. p. 169-184.

43. Gutiérrez A. Las prácticas sociales: una introducción a Pierre Bourdieu. Buenos Aires: Ferreyra Editor; 2005. 128 p.

44. Spinelli H. Las dimensiones del campo de la salud en Argentina. Salud Colect. 2010;6(3):275-293.

45. Bourdieu P. El sentido práctico. Buenos Aires: Siglo XXI Editores; 2007.

46. Erazo-Caicedo MI, Jiménez-Ruiz MC, Jiménez-Ruiz C. Empoderamiento y liderazgo femenino; su papel en la autogestión comunitaria en el corregimiento El Hormiguero - Valle del Cauca. Avanc Psicol Latinoam. 2014;32(1):149-157.

47. Juliao C. La praxeologia: una teoria de la práctica [Internet]. Bogotá: Corporación Universitaria Minuto de Dios; 2002. 324 p. Disponible en: http://repository.uniminuto.edu:8080/jspui/bitstream/10656/64 9/1/PraxeologiaUnaTeoriaDeLaPractica.pdf

48. Colombia. Ministerio de Salud y Protección Social. Resolución 429 de 2016, por medio de la cual se adopta la Política Atención Integral en Salud. Diario oficial, 49794 (feb. 22). Bogotá: Ministerio de Salud y Protección Social, 2016.

\footnotetext{
Notas

* Artículo de investigación

1 Héctor Abad Gómez (1921-1987) fue un médico salubrista antioqueño, profesor del departamento de medicina preventiva de la Facultad de Medicina de la Universidad de Antioquia, y primer director de la Escuela Nacional de Salud Pública entre 1963 y 1965 , conocido por sus propuestas de reformulación de la práctica médica, que cuestionaba, en la década de 1950, el énfasis individualista y carente de orientación social de la formación médica. Lideró reconocidos proyectos como la creación del año rural obligatorio para los médicos recién graduados, las promotoras rurales de salud, la creación de la Escuela Nacional de Salud Pública de la Universidad de Antioquia. Abad Gómez fue asesinado en Medellín en 1987, junto a otros profesores universitarios y líderes de derechos humanos en hechos atribuidos al paramilitarismo.

2 Que constituyen regímenes con coberturas más amplias en el contenido de la prestación de servicios, y que fueron mantenidos por la fuerza de negociación de sus trabajadores y agremiaciones sindicales: Ecopetrol, el magisterio, las fuerzas armadas y de Policía, los congresistas y los trabajadores del Instituto Nacional Penitenciario, Inpec.
} 